\title{
A long-range ordered array of copper tetrameric units embedded in an on- surface metal organic framework ${ }^{\circ}$
}

Cite as: J. Chem. Phys. 147, 214706 (2017); https://doi.org/10.1063/1.5004082

Submitted: 11 September 2017 . Accepted: 13 November 2017 . Published Online: 07 December 2017

Matteo Lo Cicero, Ada Della Pia, Massimo Riello, Luciano Colazzo, Francesco Sedona, (iD Maria Grazia Betti, Mauro Sambi, Alessandro De Vita, and (iD) Carlo Mariani

\section{COLLECTIONS}

AP This paper was selected as an Editor's Pick
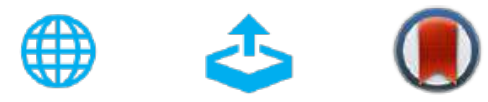

\section{ARTICLES YOU MAY BE INTERESTED IN}

Peptide dimerization-dissociation rates from replica exchange molecular dynamics

The Journal of Chemical Physics 147, 152725 (2017); https://doi.org/10.1063/1.5004774

Structure and role of metal clusters in a metal-organic coordination network determined by density functional theory

The Journal of Chemical Physics 144, 084708 (2016); https://doi.org/10.1063/1.4942665

Perspective: Size selected clusters for catalysis and electrochemistry

The Journal of Chemical Physics 148, 110901 (2018); https://doi.org/10.1063/1.5020301

\section{Challenge us.}

What are your needs for periodic signal detection?

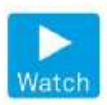

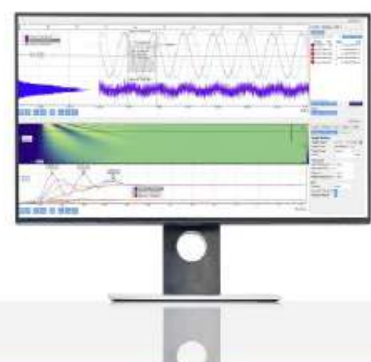

Zurich Instruments 


\title{
A long-range ordered array of copper tetrameric units embedded in an on-surface metal organic framework
}

\author{
Matteo Lo Cicero, ${ }^{1}$ Ada Della Pia, ${ }^{2}$ Massimo Riello, ${ }^{3}$ Luciano Colazzo, ${ }^{4}$ Francesco Sedona,${ }^{4}$ \\ Maria Grazia Betti, ${ }^{2}$ Mauro Sambi, ${ }^{5}$ Alessandro De Vita, ${ }^{3,6}$ and Carlo Mariani ${ }^{2, a)}$ \\ ${ }^{1}$ Dipartimento di Chimica, Università di Roma "La Sapienza," I-00185 Roma, Italy \\ ${ }^{2}$ Dipartimento di Fisica, Università di Roma "La Sapienza," I-00185 Roma, Italy \\ ${ }^{3}$ Physics Department, King's College, Strand, London WC2R 2LS, United Kingdom \\ ${ }^{4}$ Dipartimento di Scienze Chimiche, Università di Padova, Via Marzolo 1, I-35131 Padova, Italy \\ ${ }^{5}$ Dipartimento di Scienze Chimiche and INSTM, Università di Padova, Via Marzolo 1, I-35131 Padova, Italy \\ ${ }^{6}$ Department of Engineering and Architecture, University of Trieste, I-34127 Trieste, Italy
}

(Received 11 September 2017; accepted 13 November 2017; published online 7 December 2017)

\begin{abstract}
We report on the assembly of a highly ordered array of copper tetrameric clusters, coordinated into a metal-organic network. The ordered cluster array has been achieved by the deposition of tetrahydroxyquinone molecules on the $\mathrm{Cu}(111)$ surface at room temperature, and subsequent thermally activated dehydrogenation with the formation of tetraoxyquinone tetra-anions with a $4 \times 4$ periodicity. The supramolecular organic network acts as a spacer for the highly ordered two-dimensional network of copper tetramers at the very surface. Published by AIP Publishing. https://doi.org/10.1063/1.5004082
\end{abstract}

\section{INTRODUCTION}

Adsorption of single molecular units on surfaces towards fabrication of supramolecular structures is one of the most promising ways for obtaining novel two-dimensional (2D) ordered metal-organic architectures, ${ }^{1}$ with unique and peculiar structural features, functionalities, and catalytic properties..$^{2-8}$ In this way, single metal atoms embedded into an organic cage $^{9,10}$ or small metal clusters ${ }^{11,12}$ can be obtained in a highly ordered fashion within a quasi-planar 2D molecular lattice. For metals such as gold, clusters made of few atoms often present catalytic properties absent in their bulk counterpart, ${ }^{13}$ and also the chemical reduction efficiency of tetrameric copper clusters has been recently outlined. ${ }^{14}$ The wide range of applications that need a specific cluster size underlines the importance of developing novel techniques in order to enable the tuning of properties of the materials.

The variety of the existing techniques to produce clusters on a surface spans from wet-chemistry, ${ }^{15}$ to atomic beam deposition, ${ }^{16}$ to assisted chemical vapor deposition, ${ }^{17}$ to atomic cluster sources, ${ }^{14}$ to soft-landing of size-selected gas phase clusters onto supported surfaces, ${ }^{18}$ though a long-range highly ordered cluster distribution is not ensured. Electronic, optical, and magnetic properties are deeply related to the metal clusters size. In addition, recent evidences also show that a precise control of the registry between the surface-supported metal centers and the substrate, guaranteed by long-range order, can have a dramatic influence on their catalytic properties. ${ }^{19}$ Thus, controlling the cluster size and distribution acquires a pivotal role when tailoring such properties. Metal surface coordination networks to-date are typically composed of single metal atom centers or limited to trimers. ${ }^{11}$ In this work, we present

a)Electronic mail: carlo.mariani@uniroma1.it a novel method to produce size-selected copper tetramers by the employment of tetrahydroxyquinone (THQ) as the organic template obtaining a metal-organic network. The organic template serves as a mold to embed mono-dispersed copper tetrameric units of precise size and shape, in a long-range ordered network.

\section{EXPERIMENTAL AND THEORETICAL DETAILS}

Preparation and characterization of the samples have been performed under ultrahigh vacuum (UHV) conditions in two different experimental chambers, with base pressure in the low $10^{-10}$ mbar range, with the same main ancillary facilities for sample preparation.

The $\mathrm{Cu}(111)$ single crystal was cleaned by multiple cycles of $1 \mathrm{keV} \mathrm{Ar}{ }^{+}$sputtering and annealing at $\sim 725 \mathrm{~K}$ until a clean surface was obtained with large terraces, as confirmed by scanning tunneling microscopy (STM) imaging. Commercially available THQ molecules were deposited from a home-made quartz crucible evaporator held at $\sim 375 \mathrm{~K}$. No significant increase in the baseline pressure of the chamber was observed during evaporation. Degassing of the molecules was performed prior to evaporation for several hours at a temperature lower than the sublimation one. During deposition, the surface was always held at room temperature (RT), and the dehydrogenation was activated by subsequent thermal annealing. The annealing procedure was performed through a $20 \mathrm{~min}$ heating ramp up to $\sim 385 \mathrm{~K}$ followed by $4 \mathrm{~min}$ annealing at the same temperature.

The x-ray photoelectron spectroscopy (XPS) data were acquired at room temperature, at the ALOISA beamline of the synchrotron light source ELETTRA in Trieste (Italy). ${ }^{20,21}$ The photoemission data were collected by means of a hemispherical electron energy analyzer in normal emission while keeping the sample at grazing incidence $\left(\sim 4^{\circ}\right)$, with photon energies 
of $450 \mathrm{eV}$ (C 1s) and $650 \mathrm{eV}$ (O 1s). The binding energy of the core-level spectra was carefully calibrated by alignment with respect to the Fermi level of the copper substrate as the absolute reference.

STM images have been acquired with an Omicron scanning tunneling microscope (VT-STM). All of the STM measurements were executed at room temperature in constantcurrent mode using an electrochemically etched Pt-Ir tip. Typically, sample bias values between $-1 \mathrm{~V}$ and $+1 \mathrm{~V}$ have been adopted, and tunneling currents from 1 up to about $70 \mathrm{nA}$ have been used. The STM data were processed with the WSxM software. ${ }^{22}$ Fast Fourier Transform (FFT) filtering was applied to reduce the noise in the images.

Reflection High-Energy Electron Diffraction (RHEED) and Low-Energy Electron-Diffraction (LEED) measurements were performed at ALOISA and at the University of Padua, respectively, to check the formation of the $4 \times 4$ phase in the different chambers.

All Density Functional Theory (DFT) calculations and data post processing were carried out with the QuantumESPRESSO software package. ${ }^{23}$ Ultrasoft pseudopotentials $^{24}$ and the Perdew-Burke-Ernzerhof generalised gradient approximation (PBE-GGA) exchange-correlation ${ }^{25}$ were used. The wavefunction energy cutoff was set to $\sim 408 \mathrm{eV}$. The Brillouin zone was sampled at the $\Gamma$ point, and dipole correction was applied to all on-metal calculations. ${ }^{26}$ The $\mathrm{Cu}(111)$ surface was modeled by a three-layered slab with $\sim 18 \AA$ of vacuum between periodic replicas. The force convergence threshold for structure optimisation was set to $0.025 \mathrm{eV} / \AA$ (the bottom $\mathrm{Cu}$ layer was constrained to the bulk positions). Simulated STM images were rendered using the Tersoff-Hamann method. ${ }^{27}$

\section{RESULTS AND DISCUSSION}

The THQ molecules [sketched in Fig. 1(a)] deposited on the $\mathrm{Cu}(111)$ surface held at room temperature and annealed up to $\sim 385 \mathrm{~K}$ self-assemble into extended and single-domain islands, as revealed by the STM image shown in Fig. 1(b). These islands present a $4 \times 4$ periodicity, corresponding to an intermolecular distance of $10.2 \AA$, as revealed by low energy electron diffraction [Fig. 1(d)] and in agreement with the Fast Fourier Transform (FFT) of the STM image [Figs. 1(b) and 1(c)].

Individual molecular units appear in the STM images as doughnut-like protrusions [see also Fig. 2(a)], with a size

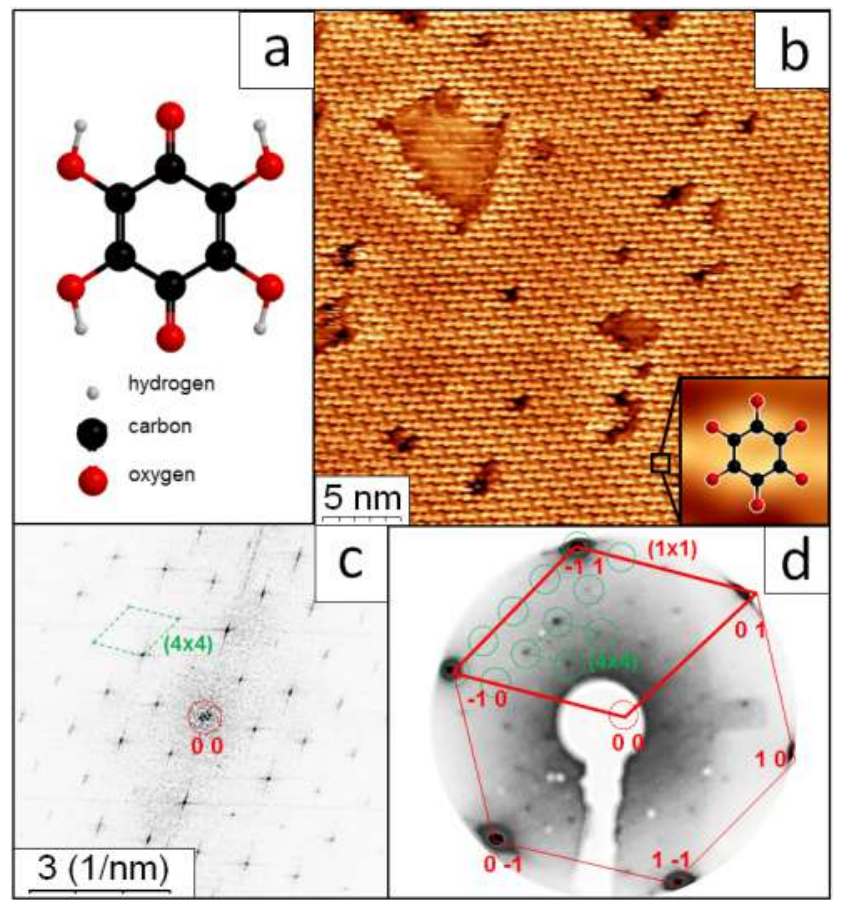

FIG. 1. (a) Ball and stick model of a THQ molecule; (b) large-scale STM image (bias $=0.55 \mathrm{~V}$; I $=37.2 \mathrm{nA}$ ) showing the self-assembly of TOQ tetraanion on $\mathrm{Cu}(111)$ after annealing to $\sim 385 \mathrm{~K}$; the TOQ tetra-anion is shown in the bottom right corner; (c) corresponding Fast Fourier Transform (FFT) image; and (d) low-energy electron-diffraction pattern acquired at an energy of $45 \mathrm{eV}$ on the same sample.

compatible with that of a single molecule and with an intermolecular distance equal to four times the interatomic copper distance. The homogeneity in the apparent height of the doughnut-like structures points toward a flat adsorption geometry of the molecules, with the molecular ring parallel to the $\mathrm{Cu}(111)$ plane. High-resolution STM images show that additional apparently triangular features are present between the doughnuts [Fig. 2(a)]. These additional features do not appear as triangles if defects are present in the molecular network, thus suggesting that the dominant triangular-shaped structure is present only when it is surrounded by three molecules (Fig. S1 of the supplementary material). These features can be associated with thermally released copper adatoms incorporated in a metal-organic network by 2,3,5,6-tetraoxyquinone tetra-anion (TOQ) units, deriving from the quadruple dehydrogenation of the THQ molecules after the annealing procedure

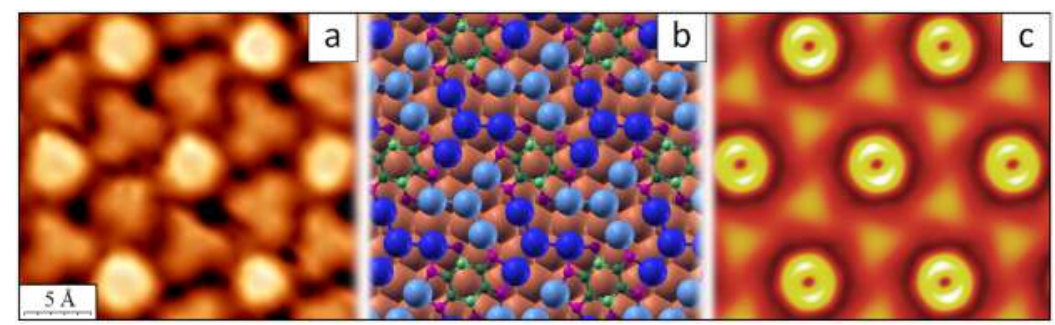

FIG. 2. (a) High-resolution STM image (bias $=0.55 \mathrm{~V}$; I = $37.2 \mathrm{nA}$ ) of the THQ tetra-anions assembled on the Cu(111) surface; (b) corresponding DFT calculated structure; and (c) simulated STM image at $0.55 \mathrm{~V}$ obtained by using the Tersoff-Hamann approach ${ }^{27}$ of the metal-organic network formed by TOQ tetra-anions on $\mathrm{Cu}(111)$ after an annealing at $\sim 385 \mathrm{~K}$. Cu adatoms are represented as light and dark blue spheres in (b) for a better visualization of the copper tetramers, while TOQ-related atoms are colored according to XPS fitting. The computed cell with parameter $10.22 \AA$ is compatible with the experimental one observed by LEED and STM. 

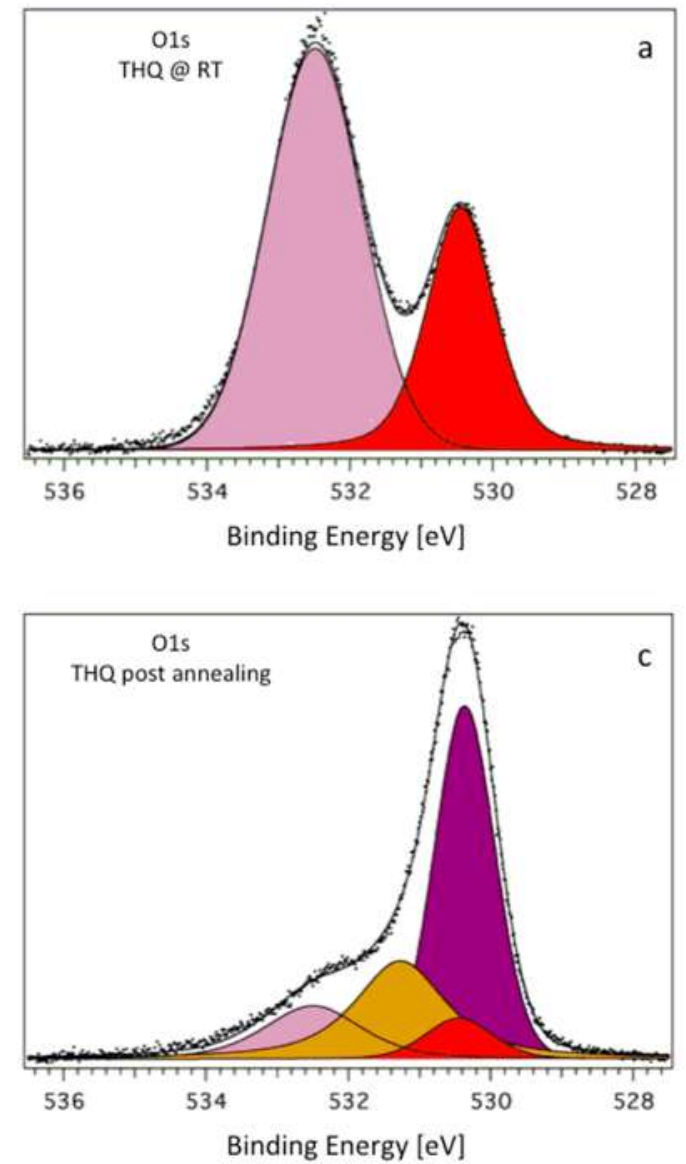
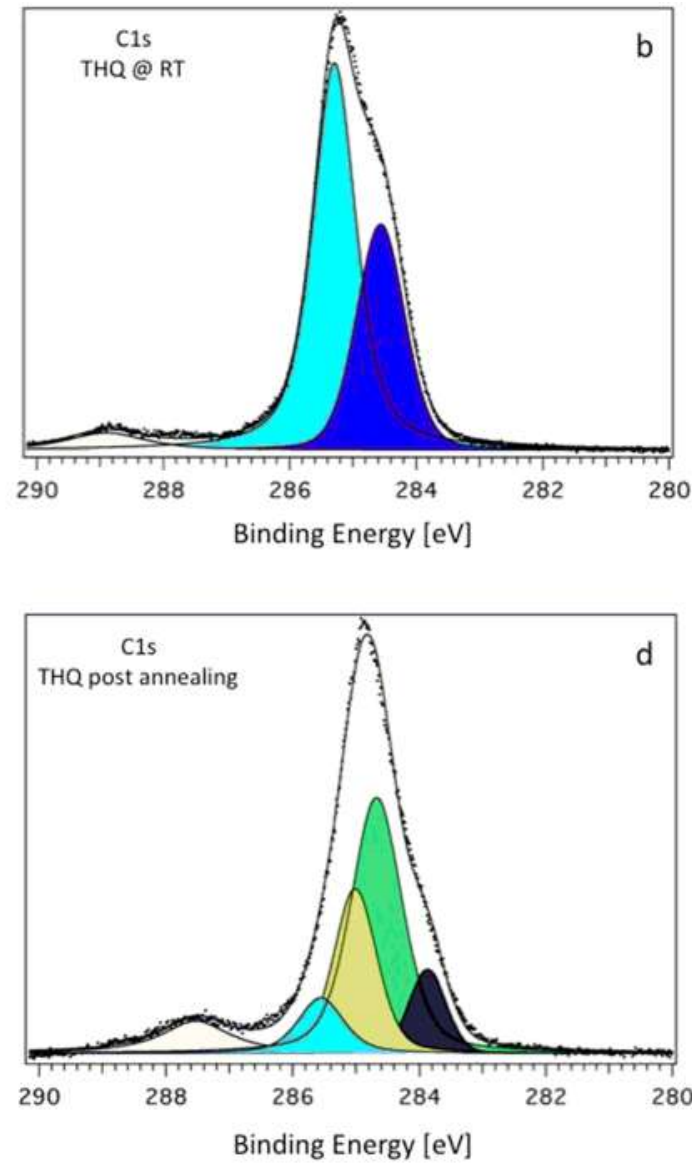

FIG. 3. O 1s (left) and C 1s (right) core level XPS measurements performed on (a) and (b) THQ molecules deposited on the Cu(111) surface at RT and (c) and (d) after an annealing at $\sim 385 \mathrm{~K}$, respectively, experimental data (dots) and results of a fitting analysis (continuous lines). The single fitting peaks are also shown and painted with the same colors of the corresponding atomic species reported in the central panel, where a sketch of the temperature-induced molecular reaction is reported.

(see molecular models in Fig. 3). The azimuthal orientation of the tetra-anions determines the formation of two different chiral assemblies, with identical TOQ adsorption sites and $4 \times 4$ LEED pattern, but a mirror azimuthal orientation for the tetra-anion and hence a mirror orientation for the tetramer (further details in Fig. S2 of the supplementary material).

Density functional theory was used to model the system shedding light on the structure of the resulting metal-organic network. Starting from the observed $4 \times 4$ structure, we carried out structural relaxations on unit cells with different THQ:Cu (adatom) ratios (see Fig. S3 of the supplementary material), in order to determine which stoichiometry best matches the experimental STM images. Our analysis identified the best ratio to be THQ: $\mathrm{Cu}$ (adatom) = 1:8 [i.e., one tetra-anion and two $\mathrm{Cu}$ tetramers per unit cell, see Fig. 2(b) and Fig. S4 of the supplementary material]. In particular, the structure in Fig. 2(b) is predicted to be energetically significantly more stable than the ones in Figs. S4 and S3 (see supplementary material) and is thus our "best" predicted structure based on theory alone. Its corresponding simulated STM image is provided in Fig. 2(c). The agreement with the experimental STM image, reported in Fig. 2(a), is very good, confirming that the triangular-shaped features observed by STM can be interpreted as tetrameric $\mathrm{Cu}$ clusters.
The ball and stick model in Fig. 2(b) illustrates how the tetrameric $\mathrm{Cu}$ clusters are formed by three peripheral adatoms that bind to $\mathrm{O}$ atoms located in TOQ tetra-anion moieties and a central adatom that does not interact with the organic medium. While the average $\sim 2.10 \AA \mathrm{Cu}-\mathrm{O}$ bonding distance is consistent with values previously reported in the literature, ${ }^{28-30}$ we find that the assembly structure contains three distinct $\mathrm{Cu}-\mathrm{O}$ bonds. Namely, two $\mathrm{O}$ atoms bound to the opposite benzene ring $\mathrm{C}$ atoms bind to a single $\mathrm{Cu}$ tetramer adatom (calculated bond length: $2.10 \AA$ ), while each of the remaining four $\mathrm{O}$ atoms in the TOQ molecule establishes two non-equivalent coordination bonds with $\mathrm{Cu}$ atoms from different tetramers (bond lengths: $2.06 \AA$ and $2.15 \AA$, respectively).

Moreover, DFT calculations confirm that the tetra-anions adsorb parallel to the substrate (Fig. S5 of the supplementary material), with the phenyl rings and the oxygen atoms lying $3.24 \AA$ and $2.99 \AA$ above the surface layer, respectively. All the adatoms constituting the tetramer unit are located at hollow surface layer sites in a geometry that alternates tetramers in hcp and fcc positions; the adsorption distance of the central adatom being slightly longer than that of the peripheral ones [2.05 $\AA$ vs. $1.99 \AA$, values close to the (111) planes' spacing]. Our DFT results provide further insights into the electronic charge redistribution within the $4 \times 4$ metal-organic network. Bader charge analysis evidenced a substantial $2.68 \mathrm{e}^{-}$ 
charge transfer from the metal surface to the TOQ tetra-anions within the resulting metal organic self-assembled adlayer. In particular, we find that each oxygen-bonded copper contributes $\sim 0.39$ a.u. to the total charge transferred to the organic medium, while the adatoms located at the center of the tetramers are practically unperturbed by the metal-organic coordination. The emerging charge rearrangement was better pointed out by calculating the differential electron charge density $\Delta \rho(\mathrm{r})$ (i.e., the difference between the charge density of the total system and the ones of the isolated tetra-anion and substrate), calculated as $\Delta \rho(\mathbf{r})=\rho_{\text {int }}(\mathbf{r})-\left[\rho_{\text {sub }}(\mathbf{r})+\rho_{\text {mol }}(\mathbf{r})\right]$.

The $\Delta \rho(\mathbf{r})$ surface plot detailed in the supplementary material (Fig. S6) reveals that the peripheral $\mathrm{Cu}$ adatoms display electron density depletion around the $\mathrm{Cu}-\mathrm{O}$ bonds and a corresponding charge accumulation on TOQ tetra-anions. While the density transferred to the tetra-anions appears to be evenly distributed over oxygens' $p$-states; the carbons' $\pi$-cloud electron density intake is not uniform. In fact, less density is accumulated in correspondence to the two equatorial $\mathrm{C}$ atoms belonging to singly coordinated carbonyls (which we will refer to as $\mathrm{C}[+] ; \mathrm{C}[-]$ will be used to indicate the remaining $4 \mathrm{C}$ atoms). The general picture of two different $\mathrm{O}$ and $\mathrm{C}$ species emerging from the calculations will be useful to interpret the core-level photoemission spectroscopy results, which we next describe.

In order to confirm the dehydrogenation process of THQ molecules upon annealing, we performed high energyresolution core-level XPS (HR-XPS) measurements (Fig. 3) on THQ molecules deposited on the $\mathrm{Cu}(111)$ surface, before and after annealing. The molecules adsorb intact onto $\mathrm{Cu}(111)$ held at room temperature. In fact, the $\mathrm{O} 1 \mathrm{~s}$ spectra display two peaks, as obtained by fitting the data with Voigt functions (Lorentzian-Gaussian lineshape, further details in the supplementary material), at $530.45 \mathrm{eV}$ and $532.50 \mathrm{eV}$ [Fig. 3(a)]. The component at lower binding energy (BE) is associated to $\mathrm{C}=\mathrm{O}$ oxygen, while the peak at a higher $\mathrm{BE}$ is associated to $\mathrm{C}-\mathrm{O}-\mathrm{H}$. Consistently, the $\mathrm{C}$ 1s core level of as-deposited THQ molecules shows two contributions at $285.30 \mathrm{eV}$ and $285.55 \mathrm{eV}$, associated with $\mathrm{C}=\mathrm{O}$ (lower $\mathrm{BE}$ ) and $\mathrm{C}-\mathrm{O}-\mathrm{H}$ (higher $\mathrm{BE}$ ) carbon, respectively, plus the corresponding shake-up peaks [Fig. 3(b)]. Previous studies using molecules similar to THQ deposited on $\mathrm{Cu}(111)$ and $\mathrm{Au}(111)$ found that at $\mathrm{RT}$, the $\mathrm{C}=\mathrm{O}$ groups point toward the substrate, which is reflected in the low $\mathrm{BE}$ of the respective $\mathrm{C} 1 \mathrm{~s}$ and $\mathrm{O}$ 1s peaks. ${ }^{11,12}$ As expected from the molecular stoichiometry for intact THQ molecules, the intensities (peak areas) of the $\mathrm{C}=\mathrm{O} / \mathrm{O}-\mathrm{H}$ related components are in a 1:2 ratio for both the $\mathrm{O} 1 \mathrm{~s}$ and $\mathrm{C} 1 \mathrm{~s}$ peaks, thus confirming the adsorption of intact molecules at RT.

Upon annealing up to $\sim 385 \mathrm{~K}$, both the $\mathrm{O} 1 \mathrm{~s}$ and $\mathrm{C} 1 \mathrm{~s}$ core level peaks change drastically [Figs. 3(c) and 3(d)]. More in detail, the $\mathrm{O} 1 \mathrm{~s} \mathrm{O}-\mathrm{H}$ associated peak disappears almost completely, indicating that most of the THQ molecules undergo a surface-assisted dehydrogenation after annealing, where copper atoms have a crucial role in the activation of the dehydrogenation process. Two main components appear in the spectrum at $530.35 \mathrm{eV}$ and $531.30 \mathrm{eV}$ BEs, with a residual small signal due to some unreacted molecules [Fig. 3(c)]. Referring to the model shown in Fig. 2(b), the peak at $530.35 \mathrm{eV}$ corresponds to oxygen atoms bound to two copper adatoms, while the peak corresponding to oxygen atoms bound to one copper adatom appears at a higher binding energy. This assignment is justified by the DFT model, suggesting that there are four oxygen atoms bound to two different copper adatoms and two oxygen atoms bound to one copper adatom only, fully consistent with the 2:1 intensity ratio in the XPS spectrum. After annealing, the $\mathrm{C}$ 1s peak is characterized by an intense component at $284.65 \mathrm{eV} \mathrm{BE}$, associated with the $\mathrm{C}[-]$ atoms, and a second one, with an area equal to half of the first one, at $285.00 \mathrm{eV} \mathrm{BE}$, corresponding to the two equatorial $\mathrm{C}[+]$ atoms. The $\mathrm{C}[-]$ atoms receive more charge from the substrate then the $\mathrm{C}[+]$ atoms, which is reflected by the lower binding energy of the corresponding peak [Fig. 3(d)]. The C 1s peak after the annealing is also characterized by a tail at low BEs that can be explained by the presence of a low amount of graphitic carbon (black component at $283.90 \mathrm{eV}$ ) due to a partial degradation of the pristine intact molecules. It is worth to note that the TOQ synthesis has been possible only thanks to surface stabilization, as the high ex-situ reactivity of such organic tetraanions prevents the possibility to directly sublimate them on the surface.

\section{CONCLUSIONS}

In conclusion, we synthesized and characterized a 2Dordered assembly of inter-linked copper tetramers through dehydrogenated THQ molecules on the $\mathrm{Cu}(111)$ surface. The ability to produce arrays of controlled, spaced tetrameric $\mathrm{Cu}$ units is potentially very interesting for applications requiring size-selected ordered arrays of metal clusters. We fully characterized the as-deposited THQ molecules on $\mathrm{Cu}(111)$ and the post-annealing 2D metal-organic tetra-anion network. STM imaging and LEED patterns provided fundamental insights about self-assembling, while detailed XPS analysis provided information on the chemical state of the molecules and their adsorption configuration, and DFT calculations were crucial for revealing the presence of copper tetrameric clusters and interpreting the spectroscopic data. This led to a fully consistent characterization of the stable linkage structure of the tetrameric units within the metal-organic salt. The proposed method to obtain a 2D ordered array of copper tetrameric units embedded in an on-surface metal-organic framework can open the perspective to apply this approach also to other molecular systems, by using ordered networks of self-assembled pristine molecular units on surfaces, followed by gentle thermal procedures, so to be able to produce size-selected metal clusters of precise size and shape in a long-range ordered array.

\section{SUPPLEMENTARY MATERIAL}

See supplementary material for additional STM images of single defects and chiral molecular assemblies, for details on the DFT calculation and on theoretical approaches towards the optimisation of THQ and the tetramer assembly, and for details on the fitting procedures. 


\section{ACKNOWLEDGMENTS}

This work was funded by Ateneo Funds of Sapienza University, MAE Grant No. US14GR12 and by the University of Padova, Grant No. CPDA154322. A.D.P. acknowledges La Sapienza University for her fellowship. We acknowledge the beamline scientists from ALOISA for support during the experiments. A.D.P. and A.D.V. acknowledge support from the EPSRC Grant No. EP/G044864/1 in developing techniques used in this work. We are grateful to the UK Materials and Molecular Modelling Hub for computational resources, which are partially funded by EPSRC (No. EP/P020194/1). A.D.V. and M.R. acknowledge funding from the European Union's Horizon 2020 research and innovation program (Grant No. 676580, The NOMAD Laboratory, a European Centre of Excellence).

${ }^{1}$ L. Dong, Z. Gao, and N. Lin, Prog. Surf. Sci. 91, 101 (2016).

${ }^{2}$ D. Ecija, S. Vijayaraghavan, W. Auwärter, S. Joshi, K. Seufert, C. Aurisicchio, D. Bonifazi, and J. V. Barth, ACS Nano 6, 4258 (2012).

${ }^{3}$ S. Stepanow, M. Lingenfelder, A. Dmitriev, H. Spillmann, E. Delvigne,

N. Lin, X. Deng, C. Cai, J. V. Barth, and K. Kern, Nat. Mater. 3, 229 (2004).

${ }^{4}$ T. Kudernac, S. Lei, J. A. A. W. Elemans, and S. De Feyter, Chem. Soc. Rev. 38, 402 (2009).

${ }^{5}$ D. Grumelli, B. Wurster, S. Stepanow, and K. Kern, Nat. Commun. 4, 2904 (2013).

${ }^{6}$ A. Dmitriev, H. Spillmann, N. Lin, J. V. Barth, and K. Kern, Angew. Chem. 115, 2774 (2003).

${ }^{7}$ M. Rosseinsky, Microporous Mesoporous Mater. 73, 15 (2004), MetalOrganic Open Frameworks.

${ }^{8}$ D. Perepichka and F. Rosei, Angew. Chem., Int. Ed. 46, 6006 (2007).

${ }^{9}$ S. Fortuna, P. Gargiani, M. G. Betti, C. Mariani, A. Calzolari, S. Modesti, and S. Fabris, J. Phys. Chem. C 116, 6251 (2012).

${ }^{10}$ M. G. Betti, P. Gargiani, C. Mariani, R. Biagi, J. Fujii, G. Rossi, A. Resta, S. Fabris, S. Fortuna, X. Torrelles, M. Kumar, and M. Pedio, Langmuir 28, 13232 (2012).

${ }^{11}$ F. Bebensee, K. Svane, C. Bombis, F. Masini, S. Klyatskaya, F. Besenbacher, M. Ruben, B. Hammer, and T. Linderoth, Chem. Commun. 49, 9308 (2013).

${ }^{12}$ F. Bebensee, K. Svane, C. Bombis, F. Masini, S. Klyatskaya, F. Besenbacher, M. Ruben, B. Hammer, and T. R. Linderoth, Angew. Chem., Int. Ed. 53, 12955 (2014).
${ }^{13}$ A. S. K. Hashmi and G. J. Hutchings, Angew. Chem., Int. Ed. 45, 7896 (2006).

${ }^{14}$ C. Liu, B. Yang, E. Tyo, S. Seifert, J. DeBartolo, B. von Issendorff, P. Zapol, S. Vajda, and L. A. Curtiss, J. Am. Chem. Soc. 137, 8676 (2015).

${ }^{15}$ T. Vossmeyer, L. Katsikas, M. Giersig, I. G. Popovic, K. Diesner, A. Chemseddine, A. Eychmueller, and H. Weller, J. Phys. Chem. 98, 7665 (1994).

${ }^{16}$ W. Benten, N. Nilius, N. Ernst, and H.-J. Freund, Phys. Rev. B 72, 045403 (2005).

${ }^{17}$ C. Navío, S. Vallejos, T. Stoycheva, E. Llobet, X. Correig, R. Snyders, C. Blackman, P. Umek, X. Ke, G. V. Tendeloo, and C. Bittencourt, Mater. Chem. Phys. 134, 809 (2012).

${ }^{18}$ X. Tang, X. Li, Y. Wang, K. Wepasnick, A. Lim, D. H. Fairbrother, K. H. Bowen, T. Mangler, S. Noessner, C. Wolke, M. Grossmann, A. Koop, G. Gantefoer, B. Kiran, and A. K. Kandalam, J. Phys.: Conf. Ser. 438, 012005 (2013).

${ }^{19}$ F. Sedona, M. Di Marino, D. Forrer, A. Vittadini, M. Casarin, A. Cossaro, L. Floreano, A. Verdini, and M. Sambi, Nat. Mater. 11, 970 (2012).

${ }^{20}$ L. Floreano, G. Naletto, D. Cvetko, R. Gotter, M. Malvezzi, L. Marassi, A. Morgante, A. Santaniello, A. Verdini, F. Tommasini, and G. Tondello, Rev. Sci. Instrum. 70, 3855 (1999).

${ }^{21}$ R. Gotter, A. Ruocco, A. Morgante, D. Cvetko, L. Floreano, F. Tommasini, and G. Stefani, in Proceedings of the 7th International Conference on Synchrotron Radiation Instrumentation [Nucl. Instrum. Methods Phys. Res., Sect. A 467, 1468 (2001)].

${ }^{22}$ I. Horcas, R. Fernández, J. M. Gómez-Rodríguez, J. Colchero, J. Gómez-Herrero, and A. M. Baro, Rev. Sci. Instrum. 78, 013705 (2007).

${ }^{23}$ P. Giannozzi, S. Baroni, N. Bonini, M. Calandra, R. Car, C. Cavazzoni, D. Ceresoli, G. L. Chiarotti, M. Cococcioni, I. Dabo, A. D. Corso, S. de Gironcoli, S. Fabris, G. Fratesi, R. Gebauer, U. Gerstmann, C. Gougoussis, A. Kokalj, M. Lazzeri, L. Martin-Samos, N. Marzari, F. Mauri, R. Mazzarello, S. Paolini, A. Pasquarello, L. Paulatto, C. Sbraccia, S. Scandolo, G. Sclauzero, A. P. Seitsonen, A. Smogunov, P. Umari, and R. M. Wentzcovitch, J. Phys.: Condens. Matter 21, 395502 (2009).

${ }^{24}$ D. Vanderbilt, Phys. Rev. B 41, 7892 (1990).

${ }^{25}$ J. P. Perdew, K. Burke, and M. Ernzerhof, Phys. Rev. Lett. 77, 3865 (1996).

${ }^{26}$ L. Bengtsson, Phys. Rev. B 59, 12301 (1999).

${ }^{27}$ J. Tersoff and D. R. Hamann, Phys. Rev. B 31, 805 (1985).

${ }^{28}$ A. Della Pia, M. Riello, J. Lawrence, D. Stassen, T. S. Jones, D. Bonifazi, A. De Vita, and G. Costantini, Chem. - Eur. J. 22, 8105 (2016).

${ }^{29}$ P. Kircher, G. Huttner, K. Heinze, B. Schiemenz, L. Zsolnai, M. Büchner, and A. Driess, Eur. J. Inorg. Chem. 1998, 703.

${ }^{30}$ R. C. Mehrotra and R. Bohra, Metal Carboxylates (Academic Press, 1983). 